Canadian Journal of Higher Education

Revue canadienne d'enseignement supérieur

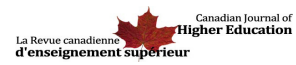

\title{
L'efficacité de la formation à distance au niveau postsecondaire : une méga-analyse
}

Yanik Simard, Clermont Gauthier and Mario Richard

Volume 49, Number 2, 2019

URI: https://id.erudit.org/iderudit/1063777ar

DOI: https://doi.org/10.7202/1063777ar

\section{See table of contents}

Publisher(s)

Canadian Society for the Study of Higher Education

ISSN

2293-6602 (digital)

Explore this journal

Cite this article

Simard, Y., Gauthier, C. \& Richard, M. (2019). L'efficacité de la formation à distance au niveau postsecondaire : une méga-analyse. Canadian Journal of Higher Education / Revue canadienne d'enseignement supérieur, 49(2), 17-36. https://doi.org/10.7202/1063777ar
Article abstract

In Canada, distance education (DE) is present in the majority of post-secondary institutions. However, there is a constant polemic in the scientific community. This is the controversial impact of technological advances on the effectiveness of this type of training. Some authors highlight the positive contribution of these advances while others emphasize the many disappointments caused by the use of newer technologies. In the face of these conflicting positions, several researchers agree that studies on the effectiveness of DE based on

technological advances are still in their infancy. To fill this gap, the objective of this research is to verify the effectiveness of the DE based on technological advances using a mega-analysis. Based on a synthesis of 16 meta-analyses involving 862 primary studies involving over 200,000 participants, the positive results obtained by our mega-analysis would support the use of more recent technologies to promote distance learning.
This document is protected by copyright law. Use of the services of Érudit (including reproduction) is subject to its terms and conditions, which can be viewed online.

https://apropos.erudit.org/en/users/policy-on-use/ 


\title{
L'efficacité de la formation à distance au niveau postsecondaire : une méga-analyse
}

\author{
Yanik Simard \\ Université TÉLUQ \\ Clermont Gauthier \\ Université Laval \\ Mario Richard \\ Université TÉLUQ
}

\section{Résumé}

Au Canada, la formation à distance (FAD) est présente dans la majorité des institutions d'enseignement postsecondaires. Cependant, une polémique revient constamment dans la communauté scientifique quant à l'incidence des avancées technologiques sur l'efficacité de ce type de formation. Certains auteurs mettent en relief l'apport positif de ces avancées tandis que d'autres insistent sur les nombreuses déceptions causées par l'utilisation des technologies plus récentes. Devant ces positions discordantes, plusieurs chercheurs conviennent que les études concernant l'efficacité de la FAD selon les avancées technologiques n'en seraient encore qu'à leurs débuts. Pour combler cette carence, l'objectif de la présente recherche est de vérifier l'efficacité de la FAD selon les avancées technologiques à l'aide d'une méga-analyse. Les résultats positifs obtenus à l'issue de notre méga-analyse, réalisée à partir d'une synthèse de 16 méta-analyses comprenant 862 études primaires auxquelles ont pris part au-delà de 200000 participants, soutiendraient le recours aux technologies plus récentes pour favoriser l'apprentissage à distance.

\footnotetext{
Abstract

In Canada, distance education (DE) is present in the majority of post-secondary
} 
institutions. However, there is a constant polemic in the scientific community. This is the controversial impact of technological advances on the effectiveness of this type of training. Some authors highlight the positive contribution of these advances while others emphasize the many disappointments caused by the use of newer technologies. In the face of these conflicting positions, several researchers agree that studies on the effectiveness of DE based on technological advances are still in their infancy. To fill this gap, the objective of this research is to verify the effectiveness of the DE based on technological advances using a mega-analysis. Based on a synthesis of 16 meta-analyses involving 862 primary studies involving over 200,000 participants, the positive results obtained by our mega-analysis would support the use of more recent technologies to promote distance learning.

L’internationalisation est une priorité pour la majorité des institutions postsecondaires canadiennes (Cui, Arthur et Domene, 2017). De même en est-il de l'accès de l'ensemble de la population du Canada à l'enseignement supérieur. Grâce notamment à la formation à distance (FAD) et à l'apprentissage en ligne, qui en constitue l'une des modalités dominantes (Bates, 2018), les universités et collèges canadiens comptent dans leurs rangs de plus en plus d'étudiants internationaux (Bozkurt, Yazıc et Aydın, 2018; Cui et al., 2017) et canadiens, dont les taux d'inscription en FAD ont connu une augmentation annuelle respective de $10 \%$ et $15 \%$ au cours des cinq dernières années (Bates, 2018).

L'avènement des nouvelles technologies n'est pas étranger à cette situation (Banerjee et Firtella, 2017; Khadimally, 2018). En effet, les technologies utilisées actuellement dans l'enseignement postsecondaire canadien sont de plus en plus variées. Si la correspondance postale est encore déployée comme moyen de diffusion, l'Internet est actuellement le principal procédé de transmission du matériel pédagogique (Bates, 2018). De plus, selon Bates, le quart des institutions postsecondaires utilisent des technologies favorisant une communication synchrone. Pour un autre quart, le matériel pédagogique est transmis électroniquement. Quant aux technologies moins dispendieuses, comme les médias sociaux et les supports mobiles, elles sont encore sous-employées.

Depuis plusieurs années, il y a une controverse dans le domaine de l'éducation, dont celui de la FAD (Collin, Guichon et Ntébutsé, 2015; Fendler, Ruff et Shrikhande, 2018; Zancaro, Nunes et Domingues, 2017), en ce qui concerne l'incidence des avancées technologiques. Certains auteurs font état de leur apport positif (Lee, 2017), tandis que d'autres insistent sur les déceptions au regard des technologies récentes (Johnson, 2014; Merrill et Ellen, 2014). Plus spécifiquement, au Canada, la résistance à l'utilisation des nouvelles technologies est évoquée dans près des deux tiers des institutions postsecondaires, et ce, malgré les priorités déclarées dans les plans quinquennaux de ces mêmes institutions (Bates, 2018). Devant ces positions discordantes, de nombreux chercheurs conviennent que les études pertinentes n'en seraient encore qu'à leurs débuts (Günes et Bosna, 2018; Kesim, 2018; Martin, Ahlgrim-Delzell et Budhrani, 2017). Il s'avère donc essentiel de mener des recherches additionnelles (Lee, 2017).

L'importance d'étudier l'efficacité de la FAD au regard des technologies anciennes ou nouvelles est également mise en relief par de nombreuses discussions sur le rôle de ces diverses technologies. Si les 203 institutions postsecondaires canadiennes consul- 
tées priorisent, dans leur plan quinquennal, l'internationalisation des apprenants ainsi qu'une accessibilité plus grande de l'enseignement supérieur pour les citoyens du pays, elles signalent, du même coup, un manque notable de fonds publics relatifs à l'intégration toujours plus intensive des nouvelles technologies (Bates, 2018). Mais, avant de pouvoir justifier de tels investissements, il importe de vérifier si l'efficacité de la FAD est vraiment plus élevée avec des technologies nouvelles.

L'objectif de notre étude est de vérifier si l'efficacité de la FAD diffère selon les avancées technologiques, c'est-à-dire selon l'utilisation de technologies anciennes ou plus récentes. Pour ce faire, nous analysons l'efficacité de la FAD au regard des technologies anciennes ou récentes, et ce, dans diverses structures internationales offrant de la FAD. Notre étude comprend des étudiants inscrits à plusieurs diverses institutions d'enseignement supérieur sur quatre continents, soit en Amérique, en Asie, en Europe ainsi qu'en Océanie.

\section{Aspects conceptuels}

La question de l'efficacité est au centre des débats entourant la FAD. Dans le cadre de notre étude, cette notion renvoie à la définition élaborée par la chercheuse Abid-Zarrouk, qui l'a appliquée au contexte de l'enseignement à distance. Pour elle, il y a l'efficacité individuelle, qui est " corrélée à la satisfaction de l'étudiant » (Pourcelot et Abid-Zarrouk, 2015, p. 3), et celle dite institutionnelle, qui correspond à l'efficacité déterminée par « le concepteur, le décideur politique [...] dans le but d'atteindre un objectif donné, décidé par lui » (Abid-Zarrouk, 2013, p. 3). Cette efficacité institutionnelle, au centre de la présente recherche, peut comprendre, selon cette chercheuse, un aspect externe ou interne. Notre étude ne porte pas sur l'efficacité externe, qui se définit comme « la mesure de la capacité éducative dans son ensemble au sein d'une institution scolaire [qui] s'apprécie à travers les perspectives d'emploi et de gains des étudiants » (Abid-Zarrouk, 2010, p. 106), mais plutôt sur l'efficacité institutionnelle interne, qui est « mesurée traditionnellement par les taux de réussite ou des résultats à des tests de connaissance » (Abid-Zarrouk, 2013, p. 3).

Pour préciser notre problématique, il faut également expliciter les théories propres à la FAD. Parmi ces théories, celles qui sont les plus citées et qui sont considérées comme les plus crédibles sont celles de Moore (Kumtepe et Ersov, 2018), d'Anderson (Carter, Solberg et Solberg, 2017) et de Garrison et Akyol (Bozkurt et al., 2015; Saba, 2013).

Dans sa théorie de la distance transactionnelle, Moore (2013) précise que la transaction est le résultat de l'interaction entre les professeurs et les apprenants se déroulant au sein de lieux spatialement séparés. La distance transactionnelle n'est pas une réalité fixe, mais variable. Elle résulte des combinaisons changeantes entre le degré de structure, de dialogue et d'autonomie de l'étudiant. Elle est très grande si le cours a une structure élevée et comprend peu de dialogue. Elle est plus petite s'il y a un dialogue intensif et une structure souple. Cependant, la pensée de Moore est peu explicite au sujet d'une variation possible de l'efficacité de la FAD selon les avancées technologiques. Pour lui, c'est la conception pédagogique qui dicte les principes de l'enseignement et de l'apprentissage, et non pas les technologies en soi. D'après Moore (2013), un dialogue (interaction) qui serait limité à de la correspondance (la poste) et au visionnement de vidéos ne favoriserait certainement pas une inscription dans une pédagogie socioconstructiviste, ou du moins le ferait de façon très limitée. Toutefois, cet auteur ne traite pas de l'efficacité de la FAD sur la base de la pédagogie déterminée par le concepteur de cours. Ainsi, Moore en arrive à 
la conclusion que plus les technologies récentes sont accessibles, plus les concepteurs de cours ont le loisir d'opter pour différents types de pédagogie.

La position qui émane de la théorie d'Anderson (2013) semble, de prime abord, un peu plus claire en ce qui concerne l'efficacité de la FAD selon les avancées technologiques. Pour cet auteur, la notion d'autodirection englobe deux dimensions, soit l'apprentissage indépendant et l'apprentissage collaboratif. Cette notion précise comment les étudiants construisent eux-mêmes leur propre milieu d'apprentissage indépendant ou collaboratif. Ils contrôlent ainsi leur mode d'acquisition des connaissances au sein d'une transaction, plus ou moins soutenue, impliquant notamment les professeurs et les pairs. Selon Anderson, la formation en ligne apporte beaucoup de possibilités d'interaction avec les acteurs et le contenu de cours grâce aux technologies récentes. En se référant à ces affirmations, on pourrait penser que la position de ce théoricien est explicite. D’après ce dernier, l'efficacité de la FAD augmenterait selon les avancées technologiques. Cependant, le point de vue d'Anderson n'est pas formulé ou explicité d'une façon aussi déterminée.

La position de Garrison et Akyol (2013) est plutôt équivoque au regard de l'efficacité de la FAD selon les avancées technologiques. Pour ces auteurs, un contexte d'apprentissage en ligne facilite grandement la mise en place d'une communauté d'apprentissage. Cependant, dans un tel contexte, l'organisation de l'enseignement est plus exigeante. Elle implique la nécessité d'intégrer la technologie au sein de la pédagogie ainsi que l'obligation de revisiter et de reconsidérer les stratégies éducatives afin de maximiser le potentiel des médias. Garrison et Akyol soulignent cette exigence de manière telle que l'on pourrait penser que l'efficacité de la FAD n'est pas nécessairement plus élevée si l'utilisation des technologies plus récentes devient incontournable. D'après ces théoriciens, force est d'admettre que la simple adoption de certaines technologies, anciennes ou nouvelles, ne résout pas tous les problèmes relatifs à l'apprentissage de la pensée critique et à la compréhension des aspects métacognitifs inhérents. Ainsi, pour ces auteurs, l'efficacité de la FAD différerait selon les avancées technologiques, mais cela ne signifierait pas que cette efficacité augmenterait avec une technologie plus avancée.

Dans l'ensemble, ces positions conceptuelles émanant des principales théories de la FAD sont relativement ambiguës au regard de notre problématique. Si l'interaction entre les apprenants est au cœur de la réussite selon ces trois conceptions, les auteurs n'indiquent pas si cette interaction est plus efficace avec l'utilisation de technologies récentes, lesquelles favorisent une interaction synchrone. Pourtant, dans l'évaluation de l'incidence des technologies sur l'apprentissage, on peut recenser deux grandes approches : une première qui soutient qu'il n'y a pas de différence significative sur les résultats des apprenants entre la FAD et l'enseignement en présentiel (Clark, 2008; Russell, 2001), et une seconde qui met de l'avant l'idée que la FAD a une incidence positive sur l'apprentissage comparativement à l'enseignement en présentiel (Fendler et al., 2018). Plus précisément, d'après Abid-Zarrouk (2012a), la première approche prétend qu'il n'y a pas de différence significative entre l'enseignement en présentiel et l'enseignement à distance lorsqu'on a recours à des technologies éducatives dites numériques. La seconde approche postule qu'au contraire, l'utilisation des technologies numériques influence positivement l'apprentissage. Ces deux approches sont prises en compte dans notre méga-analyse selon un angle particulier. En effet, cette étude vise à vérifier si l'utilisation des technologies nouvelles permet d'obtenir une différence, significative ou non, dans 
l'efficacité de la FAD lorsqu'on la compare aux technologies plus anciennes.

\section{Méthodologie et état de la recherche}

La question principale de l'étude est la suivante : l'efficacité de la FAD diffère-t-elle selon les avancées technologiques? Quant à la méthodologie choisie, elle correspond à une méga-analyse, qui se définit comme " une synthèse des résultats provenant de différentes méta-analyses » (Bissonnette, Richard, Gauthier et Bouchard, 2010). Elle est une solution efficace à la gestion des informations en provenance d'une multitude d'études. Il s'agit d'une " approche qui consiste à rassembler les données issues d'études quantitatives comparables et à les soumettre à nouveau à des analyses statistiques » (Fortin et Gagnon, 2015). Ces études, dites primaires, sont des recherches expérimentales qui produisent des données probantes portant sur une même thématique. Il faut souligner que ce sont les méta-analyses de premier degré qui se voient attribuer ici l'appellation de « méta-analyse » (Borenstein et Tanner-Smith, 2016; Landers et Reddock, 2016; Zheng, 2016). Elles consistent à rassembler des études primaires. Les méta-analyses de deuxième degré sont quant à elles basées sur des méta-analyses de premier degré. La présente recherche correspond à une méta-analyse de deuxième degré. Quant aux appellations des méta-analyses de deuxième degré, elles sont diverses en langue anglaise : meta-metaanalysis; meta-analysis of meta-analyses; network of meta-analyses; mega-analysis, etc. Notre choix s'est arrêté sur l'appellation de méga-analyse pour des raisons essentiellement pratiques. Elle est la plus succincte.

Afin de connaître l'état de la recherche, divers balayages ont été effectués (années 2000-2017) concernant les études relatives aux méga-analyses et aux méta-analyses. Les banques de données suivantes ont été consultées : Academic Search Premier; Cairn; Current Content Connect; Disssertations \& Theses Full Text Global; Education Source; ERIC; Google Scholar; PASCAL et FRANCIS en accès libre; PSYNET. L'état de la recherche montre une rareté d'études pertinentes. Dans un premier balayage, aucune mégaanalyse n'a été repérée. Dans un deuxième balayage, nous avons trouvé une quinzaine de méta-analyses, mais aucune ne faisant une distinction selon les avancées technologiques (Lee, 2017; Simard, 2018). En effet, si certaines recensions des écrits et méta-analyses portent sur l'efficacité de la $\mathrm{FAD}$, aucune d'entre elles n'offre de résultats comparant cette efficacité selon l'utilisation de technologies anciennes ou récentes. En somme, l'état des études méta-analytiques met en évidence le fait qu'une quinzaine d'entre elles se sont réellement intéressées à l'efficacité de la $\mathrm{FAD}$. Cependant, aucune de ces dernières ne présente de données relatives à son efficacité au regard des avancées technologiques. En effet, dans ces méta-analyses, aucun critère d'inclusion ou d'exclusion ne consiste expressément en des guides pour introduire ou éliminer des études primaires selon que ces dernières emploient un type quelconque de technologies. Pourtant, l'importance de procéder à de telles études a été maintes fois soulignée (Johnson, 2014; Lee, 2017; Van der Kleij et al., 2015). Donc, une méga-analyse ayant pour but de vérifier l'efficacité de la FAD selon les avancées technologiques est nécessaire.

L'une des démarches clés relatives à une méga-analyse est l'élaboration d'une classification originale des méta-analyses recensées. Notre classification comprend trois catégories d'avancées technologiques. Elle a été inspirée par plusieurs écrits, dont ceux de Richardson, Maeda, Lv et Caskurlu (2017) et de Hsu, Chibng et Grabowski (2014). Une 
première catégorie est appelée " prior distance education » $(\mathrm{PDE})^{1}$. Ces technologies plus anciennes comprennent, par exemple, la communication par correspondance postale, l'envoi de vidéos, la transmission d'émissions de télévision, les conversations téléphoniques avec le professeur (tuteur) ou avec les autres apprenants. Une seconde catégorie de technologies est appelée " online distance education » (ODE). Globalement, il s'agit de l'apprentissage en ligne, où le professeur et les apprenants demeurent isolés dans l'espace, comme dans tout autre type de FAD. Une troisième catégorie de technologies est appelée PDE/ODE, soit une combinaison de PDE et d'ODE. Dans cette catégorie, le degré d'interaction entre le professeur et les pairs est moins élevé qu'avec les ODE, mais plus élevé qu'avec les PDE. Avec cette classification, il devient possible de subdiviser la quinzaine de méta-analyses recensées. Ce faisant, la question de la recherche se traduit en trois questions opérationnelles : 1. Quelle est l'efficacité de la FAD postsecondaire qui utilise du PDE? 2. Quelle est l'efficacité de la FAD postsecondaire qui utilise du PDE/ODE? 3. Quelle est l'efficacité de la FAD postsecondaire qui utilise de l'ODE?

Une autre démarche clé d'une méga-analyse est la détermination des critères d'inclusion. Dans notre étude, les méta-analyses retenues sont basées sur les 10 critères d'inclusion suivants. Il y a celles qui : 1 . vérifient l'efficacité de la FAD, c'est-à-dire qui traitent uniquement de cette dimension de la FAD ou comprennent des résultats qui lui sont propres; 2. décrivent la $\mathrm{FAD}$ comme étant une formation offerte prioritairement à distance et nécessitant la technologie, ancienne ou nouvelle; 3. définissent l'efficacité à l'aide d'indicateurs quantitatifs validés ou reconnus par les institutions éducatives postsecondaires; 4. concernent presque exclusivement la FAD ou tiennent compte des résultats spécifiques à son efficacité; 5 . ne prennent pas en considération l'enseignement hybride (blended learning); 6. ont été publiées en 2000 ou après, dans des revues avec arbitrage, des rapports de recherche signés par des experts universitaires, ou encore, sont l'objet de thèses doctorales disponibles sur Internet; 7. précisent leur démarche méthodologique en indiquant la présence de groupes témoins et expérimentaux; l'utilisation d'un devis de recherche expérimental véritable ou quasi expérimental; les éléments nécessaires permettant un classement au niveau III de Ellis (2005), c'est-à-dire, correspondant à des données probantes à grande échelle; 8 . fournissent des informations suffisantes pour être réparties dans l'une ou l'autre des trois catégories de technologies, soit PDE, ODE et PDE/ ODE; 9. identifient clairement les études primaires utilisées, les effets d'ampleur (EA) découlant de ces dernières et le nombre de sujets concernés; 10. comprennent, chacune, un ensemble d'études primaires qui leur est propre.

Sur la base de ces dix critères d'inclusion, 16 méta-analyses sont retenues. Comme l'indique le tableau I, les méta-analyses de type PDE sont celles de Allen, Mabry, Mattrey, Bourhis, Titsworth et Burrell (2004) et de Machtmes et Asher (2000). Les méta-analyses de type ODE sont celles de Chang et Lin (2013); Cook, Levinson, Garside, Dupras, Erwin et Montori (2008); Cook, Levinson, Gardside, Dupras, Erwin et Monton (2010a.); Cook, Levinson et Garside (2010b); Darabi, Liang, Suryavanshi et Yurekli (2013); Jahng, Krug et Zhang (2007); Means, Toyama, Murphy, Bakia et Jones (2010); Roberts (2011) et Sitzmann, Kraiger, Stewart et Wisher (2006). Enfin, les méta-analyses PDE/ODE sont celles de Bernard et al. (2004); Lou, Bernard et Abrami (2006); Shachar et Neumann (2003); Williams (2006) et Zhao, Lei, Yan, Lai et Tan (2005). En somme, les 16 métaanalyses retenues totalisent 862 études primaires et au-delà de 200000 participants. 
Ces sujets proviennent de différents endroits du globe, soit les continents américain (ex. : Canada, Brésil, États-Unis), asiatique (ex. : Corée du Sud, Singapour, Taiwan), européen (ex. : Espagne, Finlande, Grèce, Italie, Royaume-Uni, Turquie) et océanien (ex. : Australie, Nouvelle-Zélande). De façon plus précise, les deux méta-analyses PDE comptent 47 études primaires et 73157 sujets. Les neuf méta-analyses ODE portent sur 345 études primaires et au-delà de 44337 sujets. Et les cinq méta-analyses PDE/ODE comprennent 470 études primaires et 81594 sujets. Quant aux indicateurs utilisés dans les 16 métaanalyses, ils relèvent de l'efficacité institutionnelle (plus spécifiquement, de l'efficacité interne), et non de l'efficacité individuelle (Abid-Zarrouk, 2015; Pourcelot et Abid-Zarrouk, 2015). En effet, les indicateurs concernent tous la performance académique, c'està-dire le degré de réussite déterminé par les responsables institutionnels de l'évaluation de l'apprentissage.

Le modèle de traitement des données est l'évaluation des effets d'ampleur à l'aide d'un indicateur, c'est-à-dire d'un effet d'ampleur prédéterminé. Ce modèle est emprunté à Bissonnette et al. (2010). Leur publication est citée dans une dizaine de revues internationales. Ces auteurs n'utilisent qu'un seul point d'appui, soit un EA de 0,40. Dans notre méga-analyse, il y a un triple indicateur déterminé par Hattie (2015) et repris par plusieurs méta-analyses récentes (Sung, Chang et Liu, 2016; van der Kleij, Feskens et Eggen, 2015; Ziegler, 2016). Ce triple indicateur correspond à trois effets d'ampleur : 0,20; 0,40; 0,60. Avec ce triple indicateur, comparativement à l'enseignement en présentiel, l'efficacité de la FAD est estimée comme étant : légèrement $(\mathrm{L})$ plus élevée $(0,20)$; modérément $(\mathrm{M})$ plus élevée $(0,40)$ ou grandement $(\mathrm{G})$ plus élevée $(0,60)$. L'efficacité de la FAD est également désignée comme pouvant être inférieure (I) $(\leq-0,19)$ ou à tout le moins semblable (S) $(\geq-0,19)$.

Les 16 méta-analyses présentent des effets d'ampleur qui permettent de déterminer cette efficacité en la comparant à celle de l'enseignement en présentiel, mais ce n'est pas le but de notre méga-analyse. Pour établir si l'efficacité de la FAD diffère selon les avancées technologiques, une autre étape de traitement des données doit donc être effectuée. Il s'agit du regroupement des indicateurs d'effets d'ampleur. Ainsi, nous avons procédé à divers regroupements des indicateurs (G, M, L, S, I). De cette manière, l'efficacité de la FAD est considérée comme étant :

- à tout le moins modérément plus élevée si les effets d'ampleur sont $\geq 0,40(\mathrm{M}$ et $\mathrm{G})$;

- à tout le moins légèrement plus élevée si les effets d'ampleur sont $\geq 0,20$ (L, M et $\mathrm{G})$;

- à tout le moins semblable si les effets d'ampleur sont $\geq-0,19$ (S, M, L et G);

- inférieure si les effets d'ampleur sont $\leq-0,19$ (I).

En calculant le nombre (brut et \%) d'effets d'ampleur qui se retrouvent dans chacun de ces regroupements selon que la FAD intègre l'une ou l'autre des trois catégories (PDE, $\mathrm{PDE} / \mathrm{ODE}$ ou ODE), on peut constater si l'efficacité de la FAD diffère selon les avancées technologiques. 
Tableau 1. Les informations globales relatives aux méta-analyses retenues

\begin{tabular}{|c|c|c|c|c|c|c|c|c|}
\hline Méta-analyse & $\begin{array}{c}\text { Catégorie } \\
\text { de } \\
\text { technologie }\end{array}$ & $\begin{array}{l}\text { Année } \\
\text { de } \\
\text { publica- } \\
\text { tion }\end{array}$ & $\begin{array}{c}\text { Niveau } \\
\text { de } \\
\text { formation }\end{array}$ & $\begin{array}{c}\text { Nombre } \\
\text { de } \\
\text { sujets }\end{array}$ & $\begin{array}{l}\text { Nombre } \\
\text { d'études } \\
\text { primaires }\end{array}$ & $\begin{array}{c}\text { Nombre } \\
\text { d'effets } \\
\text { d'ampleur }\end{array}$ & $\begin{array}{l}\text { Études primaires } \\
\text { avec groupes té- } \\
\text { moins } \\
\text { et expérimentaux }\end{array}$ & $\begin{array}{l}\text { Niveau } \\
\text { hiérar- } \\
\text { chique } \\
\text { (Ellis, } \\
\text { 2005) }\end{array}$ \\
\hline Allen et al. & PDE & 2004 & postsec. & 71731 & 28 & 39 & $\mathrm{X}$ & III \\
\hline Bernard et al. & PDE/ODE & 2004 & $\begin{array}{l}\text { tous niveaux + } \\
\text { postsec. }\end{array}$ & $\begin{array}{c}35365 \\
\text { tous niveaux; } \\
\text { ND (postsec.) }\end{array}$ & $\begin{array}{c}232 \\
\text { tous niveaux; } \\
\text { ND (postsec.) }\end{array}$ & 255 (postsec.) & $\mathrm{X}$ & III \\
\hline Chang et Lin. & ODE & 2013 & $\begin{array}{l}\text { tous niveaux + } \\
\text { postsec. }\end{array}$ & 1892 (postsec.) & 19 (postsec.) & 19 (postsec.) & $\mathrm{X}$ & III \\
\hline Cook et al. & ODE & 2008 & postsec. & 7632 & 81 & 81 & $\mathrm{X}$ & III \\
\hline Cook et al. & ODE & $2010 a$ & postsec. & 4683 & 32 & 32 & $\mathrm{X}$ & III \\
\hline Cook et al. & ODE & $2010 b$ & postsec. & 808 & 8 & 8 & $\mathrm{X}$ & III \\
\hline Darabi et al. & ODE & 2013 & postsec. & 595 & 5 & 45 & $\mathrm{X}$ & III \\
\hline Jahng et al. & ODE & 2007 & postsec. & 1617 & 20 & 20 & $\mathrm{X}$ & III \\
\hline Lou et al. & PDE/ODE & 2006 & postsec. & 25230 & 103 & 218 & $\mathrm{X}$ & III \\
\hline $\begin{array}{l}\text { Machtmes et } \\
\text { Asher }\end{array}$ & PDE & 2000 & postsec. & 1426 & 19 & 19 & $\mathrm{X}$ & III \\
\hline Means et al. & ODE & 2010 & postsec. & $>2000$ & 28 & 44 & $\mathrm{X}$ & III \\
\hline Roberts & ODE & 2011 & postsec. & 5779 & 59 & 86 & $\mathrm{X}$ & III \\
\hline $\begin{array}{l}\text { Shachar et } \\
\text { Neumann }\end{array}$ & PDE/ODE & 2003 & postsec. & 15346 & 86 & 86 & $\mathrm{X}$ & III \\
\hline Sitzmann et al. & ODE & 2006 & postsec. & 19331 & 93 & 93 & $\mathrm{X}$ & III \\
\hline Williams & PDE/ODE & 2006 & postsec. & 2742 & 25 & 34 & $\mathrm{X}$ & III \\
\hline Zhao et al. & PDE/ODE & 2005 & $\begin{array}{l}\text { tous niveaux }+ \\
\text { postsec. }\end{array}$ & 2911 (postsec.) & $\begin{array}{l}24 \text { (postsec.) } \\
(20+4)\end{array}$ & $\begin{array}{l}71 \text { (postsec.) } \\
(35+36)\end{array}$ & $\mathrm{X}$ & III \\
\hline $\begin{array}{l}\text { total : } \\
16 \\
\text { méta-analyses }\end{array}$ & & & & $\begin{aligned} & \text { total : } \\
> & 200 \text { ooo } \\
& \text { sujets }\end{aligned}$ & $\begin{array}{l}\text { total : } \\
862 \text { études } \\
\text { primaires }\end{array}$ & $\begin{array}{c}\text { total : } \\
1150 \text { effets } \\
\text { d'ampleur }\end{array}$ & & \\
\hline
\end{tabular}




\section{Résultats}

Il semble bien que l'efficacité de la FAD soit plus élevée au fur et à mesure que sont utilisées les technologies plus récentes. Pour plus de précisions, voyons les six tableaux suivants. Le tableau 2 présente les méta-analyses qui utilisent la catégorie de technologies PDE. La variation des deux effets d'ampleur relevés va de -0,0093 à 0,048. Étant donné que les deux (2/2 ou $100 \%)$ effets d'ampleur se retrouvent dans le regroupement d'indicateurs $\geq-0,19$, on peut conclure que la FAD est aussi efficace que l'enseignement en présentiel lorsqu'elle fait appel à des technologies anciennes.

Tableau 2. L'efficacité de la FAD selon la catégorie de technologies PDE

\begin{tabular}{|c|c|c|c|c|c|}
\hline $\begin{array}{c}\text { Méta analyses : } \\
\text { auteurs }\end{array}$ & n. & $\begin{array}{c}\text { Effet } \\
\text { d'ampleur } \\
\text { (EA) }\end{array}$ & $\begin{array}{c}\text { Effet } \\
\text { d'ampleur } \\
\geq \mathbf{- 0 , 1 9} \\
(\mathrm{S}, \mathrm{L}, \mathrm{M} \text { ou } \mathrm{G})\end{array}$ & $\begin{array}{c}\text { Effet } \\
\text { d'ampleur } \\
\geq \mathbf{0 , 2 0} \\
(\mathrm{L}, \mathrm{M} \text { ou } \mathrm{G})\end{array}$ & $\begin{array}{c}\text { Effet } \\
\text { d'ampleur } \\
\geq \mathbf{0 , 4 0} \\
(\mathrm{M} \text { ou } \mathrm{G})\end{array}$ \\
\hline $\begin{array}{l}\text { Allen et al. } \\
\text { (2004) }\end{array}$ & 1 & 0,0480 & $\mathrm{~S}$ & & \\
\hline $\begin{array}{l}\text { Machtmes et } \\
\text { Asher (2000) }\end{array}$ & 1 & $-0,0093$ & $\mathrm{~S}$ & & \\
\hline $\begin{array}{l}\text { Total } \\
\text { des EA }\end{array}$ & 2 & & $\begin{array}{c}2 / 2 \\
(100 \%)\end{array}$ & $\begin{array}{c}0 / 2 \\
(0 \%)\end{array}$ & $\begin{array}{c}0 / 2 \\
(0 \%)\end{array}$ \\
\hline
\end{tabular}

Selon le tableau 3, les méta-analyses classées dans la catégorie des technologies PDE/ODE présentent sept effets d'ampleur qui varient de -0,0048 à 0,366. Deux effets d'ampleur (2/7 ou $29 \%$ ) se situent dans le regroupement des effets d'ampleur ayant une valeur $\geq 0,20$. Aucun effet d'ampleur n'a une valeur $\geq 0,40$. Sept effets d'ampleur (7/7 ou $100 \%$ ) se situent dans le regroupement des effets d'ampleur correspondant à une valeur $\geq-0,19$.

Tableau 3. L'efficacité de la FAD selon la catégorie de technologies PDE/ODE

\begin{tabular}{|c|c|c|c|c|c|}
\hline $\begin{array}{l}\text { Méta-analyses : } \\
\text { auteurs }\end{array}$ & $\begin{array}{c}\text { n. } \\
\mathbf{E A}\end{array}$ & $\begin{array}{c}\text { Effet } \\
\text { d'ampleur } \\
\text { (EA) }\end{array}$ & $\begin{array}{c}\text { Effet } \\
\text { d'ampleur } \\
\geq \mathbf{- 0 , 1 9} \\
(\mathrm{S}, \mathrm{L}, \mathrm{M} \text { ou } \mathrm{G})\end{array}$ & $\begin{array}{c}\text { Effet } \\
\text { d'ampleur } \\
\geq \mathbf{0 , 2 0} \\
(\mathrm{L}, \mathrm{M} \text { ou G) }\end{array}$ & $\begin{array}{c}\text { Effet } \\
\text { d'ampleur } \\
\geq \mathbf{0 , 4 0} \\
(\mathrm{M} \text { ou } \mathrm{G})\end{array}$ \\
\hline $\begin{array}{l}\text { Bernard et al. } \\
\quad(2004)\end{array}$ & 2 & $\begin{array}{l}-0,0048 \\
0,0809\end{array}$ & $\begin{array}{l}\mathrm{S} \\
\mathrm{S}\end{array}$ & & \\
\hline Lou et al. (2006) & 1 & 0,0160 & S & & \\
\hline $\begin{array}{l}\text { Shachar et Neu- } \\
\text { mann (2003) }\end{array}$ & 1 & 0,3660 & $\mathrm{~L}$ & $\mathrm{~L}$ & \\
\hline Williams (2006) & 1 & 0,1500 & S & & \\
\hline $\begin{array}{l}\text { Zhao et al. } \\
\text { (2005) }\end{array}$ & 2 & $\begin{array}{l}0,3600 \\
0,0300\end{array}$ & $\begin{array}{l}\mathrm{L} \\
\mathrm{S}\end{array}$ & $\mathrm{L}$ & \\
\hline $\begin{array}{c}\text { Total } \\
\text { des EA }\end{array}$ & 7 & & $\begin{array}{c}7 / 7 \\
(100 \%) \\
\end{array}$ & $\begin{array}{c}2 / 7 \\
(29 \%) \\
\end{array}$ & $\begin{array}{c}0 / 7 \\
(0 \%) \\
\end{array}$ \\
\hline
\end{tabular}


Selon le tableau 4, les méta-analyses utilisant les technologies ODE comprennent des effets d'ampleur dont la variation va de -0,106 à 0,856 . Sept effets d'ampleur sur un total de 16 (7/16 ou $44 \%$ ), ayant une valeur $\geq 0,20$, révèlent que, dans ces cas, l'efficacité de la FAD est plus élevée que celle du présentiel. Il en est de même avec les cinq effets d'ampleur sur un ensemble de $16(5 / 16$ ou $31 \%)$ qui ont une valeur $\geq 0,40$. La totalité des 16 effets d'ampleur (16/16 ou $100 \%$ ), ayant une valeur $\geq-0,19$, montre que l'efficacité de la FAD est, à tout le moins, semblable à celle du présentiel.

Tableau 4. L'efficacité de la FAD selon la catégorie de technologies ODE

\begin{tabular}{|c|c|c|c|c|c|}
\hline $\begin{array}{c}\text { Méta-analyses : } \\
\text { auteurs }\end{array}$ & n. & $\begin{array}{c}\text { Effet } \\
\text { d'ampleur } \\
\text { (EA) }\end{array}$ & $\begin{array}{c}\text { Effet } \\
\text { d'ampleur } \\
\geq \mathbf{- 0 , 1 9} \\
\text { (S, L, M ou G) }\end{array}$ & $\begin{array}{c}\text { Effet } \\
\text { d'ampleur } \\
\geq \mathbf{0 , 2 0} \\
(\mathrm{L}, \mathrm{M} \text { ou } \mathrm{G})\end{array}$ & $\begin{array}{c}\text { Effet } \\
\text { d'ampleur } \\
\geq \mathbf{0 , 4 0} \\
(\mathrm{M} \text { ou } \mathrm{G})\end{array}$ \\
\hline $\begin{array}{c}\text { Chang et Lin } \\
\text { (2013) }\end{array}$ & 2 & $\begin{array}{l}0,851 \\
0,677\end{array}$ & $\begin{array}{l}\mathrm{G} \\
\mathrm{G}\end{array}$ & $\begin{array}{l}\mathrm{G} \\
\mathrm{G}\end{array}$ & $\begin{array}{l}\mathrm{G} \\
\mathrm{G}\end{array}$ \\
\hline $\begin{array}{l}\text { Cook et al. } \\
(2008)\end{array}$ & 3 & $\begin{array}{l}0,120 \\
0,090 \\
0,510\end{array}$ & $\begin{array}{l}\mathrm{S} \\
\mathrm{S} \\
\mathrm{M}\end{array}$ & M & M \\
\hline $\begin{array}{l}\text { Cook et al. } \\
\text { (2010a) }\end{array}$ & 1 & 0,270 & $\mathrm{~L}$ & $\mathrm{~L}$ & \\
\hline $\begin{array}{c}\text { Cook et al. } \\
(2010 \mathrm{~b})\end{array}$ & 1 & $-0,100$ & $\mathrm{~S}$ & & \\
\hline $\begin{array}{l}\text { Darabi et al. } \\
\text { (2013) }\end{array}$ & 2 & $\begin{array}{l}0,547 \\
0,856\end{array}$ & $\begin{array}{l}M \\
G\end{array}$ & $\begin{array}{l}M \\
G\end{array}$ & $\begin{array}{l}\mathrm{M} \\
\mathrm{G}\end{array}$ \\
\hline $\begin{array}{c}\text { Jahng et al. } \\
\text { (2007) }\end{array}$ & 3 & $\begin{array}{c}0,023 \\
-0,106 \\
0,211\end{array}$ & $\begin{array}{l}\text { S } \\
\text { S } \\
\text { L }\end{array}$ & $\mathrm{L}$ & \\
\hline $\begin{array}{l}\text { Means et al. } \\
\text { (2010) }\end{array}$ & 1 & 0,140 & $\mathrm{~S}$ & & \\
\hline Roberts (2011) & 1 & 0,078 & $\mathrm{~S}$ & & \\
\hline $\begin{array}{l}\text { Sitzmann et al. } \\
\text { (2006) }\end{array}$ & 2 & $\begin{array}{c}0,150 \\
-0,070\end{array}$ & $\begin{array}{l}\mathrm{S} \\
\mathrm{S}\end{array}$ & & \\
\hline $\begin{array}{c}\text { Total } \\
\text { des EA }\end{array}$ & 16 & & $\begin{array}{c}16 / 16 \\
(100 \%)\end{array}$ & $\begin{array}{c}7 / 16 \\
(44 \%)\end{array}$ & $\begin{array}{c}5 / 16 \\
(31 \%)\end{array}$ \\
\hline
\end{tabular}

Selon le tableau 5, qui présente les principales données des tableaux 2, 3 et 4, l'efficacité de la FAD est plus élevée si elle utilise les ODE. Ainsi, l'efficacité de la FAD diffère selon les avancées technologiques, car il y a une augmentation du nombre d'effets d'ampleur $\geq$ 0,20 . En effet, le \% d'effets d'ampleur de cette valeur $\geq 0,20$ est de $0 \%$ avec les technologies PDE; $29 \%$ avec les PDE/ODE; et $44 \%$ avec les ODE. 
Tableau 5. Synthèse des résultats comparatifs de l'efficacité de la FAD selon les avancées technologiques : la proportion ou le pourcentage des effets d'ampleur $\geq 0,2 O$

$\begin{array}{cc}\text { Catégorie de technologies } & \text { Effets d'ampleu } \\ \text { PDE } & 0 / 2 \\ & (0 \%) \\ \text { PDE/ODE } & 2 / 7 \\ & (29 \%) \\ \text { ODE } & 7 / 16 \\ & (44 \%)\end{array}$

Selon le tableau 6, qui fournit les principales données des tableaux 2, 3 et 4, l'efficacité de la FAD est plus élevée si elle utilise les PDE/ODE et les ODE. Ainsi, l'efficacité de la FAD diffère selon les avancées technologiques, car il y a une augmentation du nombre d'effets d'ampleur $\geq 0,40$. En effet, le pourcentage d'effets d'ampleur de cette valeur $\geq$ 0,40 est de $0 \%$ avec les technologies PDE; o \% avec les PDE/ODE; et $31 \%$ avec les ODE.

Tableau 6. Synthèse des résultats comparatifs de l'efficacité de la FAD selon les avancées technologiques : la proportion ou le pourcentage des effets d'ampleur $\geq 0,40$

\begin{tabular}{cc}
\hline Catégorie de technologies & Effets d'ampleur $\geq \mathbf{0 , 4 0}$ \\
PDE & $0 / 2$ \\
& $(0 \%)$ \\
PDE/ODE & $0 / 7$ \\
& $(0 \%)$ \\
ODE & $5 / 16$ \\
& $(31 \%)$ \\
\hline
\end{tabular}

Enfin, selon le tableau 7, qui montre les principales données des tableaux 2, 3 et 4, l'efficacité de la $\mathrm{FAD}$ est à tout le moins semblable à celle de l'enseignement en présentiel, et ce, quelle que soit la catégorie de technologies utilisée. En effet, le pourcentage d'effets d'ampleur de cette valeur $\geq-0,19$ est de $100 \%$ avec les technologies PDE; $100 \%$ avec les PDE/ODE; et $100 \%$ avec les ODE. En somme, l'efficacité de la FAD diffère au fil des avancées technologiques. Elle montre une amélioration constante et toujours plus élevée plus les technologies sont récentes. Avec un indicateur d'une valeur $\geq 0,20$, le pourcentage des effets d'ampleur est plus grand avec les ODE (44\%) qu'avec les PDE/ODE (29\%) et les $\mathrm{PDE}$ (0 \%). Avec un indicateur d'une valeur $\geq 0,40$, le pourcentage des effets d'ampleur est plus grand avec les ODE (31 \%) qu'avec les PDE/ODE (o \%) et les PDE (o \%). 
Tableau 7. Synthèse des résultats comparatifs de l'efficacité de la FAD selon les avancées technologiques : la proportion ou le pourcentage des effets d'ampleur $\geq-0,19$

$\begin{array}{cc}\text { Catégorie de technologies } & \text { N. d'effets d'ampleur } \geq \mathbf{- 0 . 1 9} \\ \text { PDE } & 2 / 2 \\ & (100 \%) \\ \text { PDE/ODE } & 7 / 7 \\ & (100 \%) \\ \text { ODE } & 16 / 16 \\ & (100 \%)\end{array}$

\section{Discussion des résultats}

La question de l'efficacité en FAD renvoie, rappelons-le, à « deux approches portant sur l'évaluation des technologies dans l'apprentissage » (Abid-Zarrouk, 2012a, p. 2). Il y a celle qui postule l'absence d'une différence significative, dans laquelle s'inscrivent notamment les travaux de Clark (2008) et de Russell (2001), puis celle qui postule une portée positive et reconnaît un apport significatif à l'introduction des technologies. Les résultats de notre méga-analyse semblent contribuer à la confirmation de la seconde approche, mise en relief, encore tout récemment, par Fendler et al. (2018).

Par ailleurs, nos résultats apportent une certaine contribution au regard des trois principales conceptions de la FAD. Tout d'abord, dans sa notion de distance transactionnelle, Moore ne se prononce pas sur l'efficacité de la FAD selon les avancées technologiques au regard de la distance transactionnelle, grande ou petite. À partir des résultats de notre méga-analyse, nous pouvons apporter deux précisions complémentaires à la théorie de Moore : premièrement, une distance transactionnelle, même grande, permet à la FAD d'obtenir une efficacité semblable à celle du présentiel, et ce, quand elle est réalisée à l'aide de stratégies pédagogiques spécifiques faisant appel à des technologies anciennes; deuxièmement, une distance transactionnelle petite, obtenue à l'aide de stratégies pédagogiques intégrant des technologies récentes, permet à la FAD d'obtenir une efficacité plus élevée. De la même façon, Anderson, dans sa théorie de l'apprentissage indépendant, ne se prononce pas sur l'efficacité de la FAD selon les avancées technologiques. Or, à partir des résultats de notre méga-analyse, il est possible de proposer l'ajout suivant : l'efficacité de la FAD est plus élevée si elle utilise des stratégies pédagogiques relatives à l'apprentissage indépendant, et ce, à l'aide de technologies récentes. D'ailleurs, selon la méta-analyse de Zheng (2016), la majorité des cours offerts en FAD (computer-based learning), dont l'objectif est le développement de l'apprentissage indépendant (self-regulated learning), comprennent des programmes de soutien (self-regulated scaffolds). De surcroitt, plus ces programmes s'appuient sur des technologies récentes, plus ils sont aidants. D’après la théorie de la communauté d'apprentissage de Garrison et Akyol, l'efficacité de la FAD pourrait ne pas différer avec les technologies récentes étant donné la difficulté de leur intégration et l'obligation possible de modifier certaines approches pédagogiques. Or, nos résultats montrent plutôt que dans les communautés d'apprentissage, les acteurs de la FAD relèvent mieux les défis inhérents à leur apprentissage avec l'utilisation des technologies récentes. 
Concernant la méthodologie utilisée, il est possible de s'interroger sur l'effet de l'étalement dans le temps des années de publication des méta-analyses recensées sur la validité des résultats. La date de parution des méta-analyses utilisant les technologies PDE se situe entre 2000 et 2004 . Celle des méta-analyses empruntant les technologies PDE/ODE se situe entre 2003 et 2006, et celle des méta-analyses intégrant les technologies ODE se situe entre 2006 et 2013. À première vue, les données de la FAD utilisant des technologies PDE sont inexistantes au milieu de la deuxième décennie du 21e siècle. Il en va de même pour la FAD relevant de la catégorie ODE au début de la première décennie. Cependant, en y regardant de plus près, cet étalement dans le temps n'enlève rien à la validité des résultats. Ainsi, peu importe s'il s'agit d'une publication se situant dans la première ou la deuxième décennie du 21e siècle, cette situation ne change pas l'essentiel de la réponse obtenue à la question principale ou complémentaire de notre méga-analyse. En effet, dans les 16 méta-analyses retenues dans notre étude, l'efficacité de la FAD est toujours déterminée en la comparant à l'enseignement en présentiel de la même époque. Il faut noter que les trois catégories de technologie sont toujours contemporaines. En effet, encore aujourd'hui, certaines institutions offrant de la FAD ont des programmes pour les étudiants qui n'ont pas accès aux nouvelles technologies (c'est le cas par exemple de la Open University, au Royaume-Uni), ou pour les apprenants qui ne veulent pas parfaire leur formation avec ces nouvelles technologies (comme à la University of South Dakota, pour certains groupes d'infirmières en perfectionnement continu). Par ailleurs, il faut noter la conclusion d'un expert qui a traité plus de 1500 méta-analyses en éducation. D’après ce dernier, les méta-analyses semblent le plus souvent adopter une perspective transversale (synchronique), et ce, même si elles sont exclusivement axées sur le passé (Hattie, 2015). Cependant, pour cet auteur, le degré auquel ces études antérieures peuvent ou non éclairer l'éducation d'aujourd'hui ou de demain est matière à interprétation de la part du praticien, théoricien ou chercheur.

Il faut également noter que, dans notre étude, nous avons pris en compte les trois principales critiques adressées aux méga-analyses. Tout d'abord, une première critique est qu'elles sont source d'erreurs (Borenstein et Tanner-Smith, 2016). Dans notre mégaanalyse, deux démarches importantes ont été réalisées pour diminuer sensiblement les sources d'erreur. D'une part, nous n'avons inclus que les méta-analyses dont les études primaires utilisent un design de recherche rigoureux. D'autre part, nous n'avons retenu que des données probantes et à grande échelle. Une seconde critique au sujet des méga-analyses est qu'elles sont une combinaison d'études disparates. Pour éviter une telle situation, les 16 méta-analyses retenues sont uniquement celles qui ont tenu compte de l'effet d'hétérogénéité et qui ont exclu toutes les études primaires dénotant une disparité trop élevée. Ainsi, chacune des 16 méta-analyses retenues rapporte des effets d'ampleur dits consistants (expression de Borenstein et Tanner-Smith, 2016), où il n'y a aucune combinaison d'études disparates. Une troisième critique porte sur le choix du traitement des données. Rappelons-le, la procédure retenue dans notre étude est le modèle de l'évaluation des effets d'ampleur à l'aide d'indicateurs. Ce choix correspond au rejet de trois autres options nettement moins appropriées. Une première option qui a été rejetée est celle du retour aux données brutes. Ce retour implique la réutilisation des effets d'ampleur de chaque étude primaire incluse dans une méta-analyse. Si ce retour est considéré comme le procédé par excellence, il n'est pas plus efficace que l'utilisation des effets d'ampleur produits par les méta-analyses elles-mêmes (Sung et al., 2014). De plus, 
il aurait été complexe à appliquer, car il aurait fallu revoir 862 études primaires. La deuxième option qui a été écartée est le simple calcul de la moyenne des effets d'ampleur des méta-analyses. Cette procédure est très utilisée, mais aussi très critiquée. La variance des résultats est très peu prise en compte. Donc, cette option aurait présenté des résultats moins précis. La troisième option qui a été rejetée est l'utilisation de calculs statistiques encore inédits. Ces calculs seraient plus sophistiqués que ceux qui sont utilisés à l'heure actuelle, cependant, ils ne sont pas encore validés (Gurevitch, Koricheva, Nakagawa et Stewart, 2018; Schmidt et Hunter, 2015). Donc, il était nettement préférable de s'en tenir au choix de notre procédure du traitement des données.

Quant aux applications pratiques, notre méga-analyse portant sur plus de 200000 apprenants en provenance des continents américain, asiatique, européen et océanien met en lumière le fait que le recours aux technologies les plus récentes est lié à une efficacité plus élevée de la $\mathrm{FAD}$ postsecondaire. Si la $\mathrm{FAD}$ postsecondaire canadienne vise à intensifier l'internationalisation de ses structures ainsi qu'à accroître l'accès à l'enseignement supérieur pour les citoyens du pays (Bates, 2018), il faudrait conséquemment intensifier l'utilisation des nouvelles technologies. Il reste cependant à savoir dans quelles conditions cette intensification doit s'effectuer. Des recherches ultérieures sont nécessaires pour répondre à ces interrogations. Mais dans l'attente de ces réponses, les structures canadiennes de l'enseignement supérieur offertes par le biais de la FAD doivent, selon nous, considérer l'intégration des technologies récentes.

Finalement, la poursuite d'enquêtes relatives à l'efficacité de la FAD selon les avancées technologiques apparaît cruciale compte tenu de l'évolution rapide de ces dernières (Adnan, Kalilioglu, Gulbahar et Brunner, 2017; Zancaro et al., 2017). De nouvelles méga-analyses sont nécessaires pour déterminer les autres variables modératrices liées à ces avancées (Collin et al., 2015; Richardson et al., 2017). La présente méga-analyse s'avère un début, mais plusieurs questions se posent, notamment au regard des stratégies pédagogiques, des contenus de cours et des acteurs centraux de la FAD que sont les professeurs, les tuteurs et les apprenants. D'une façon plus précise, des recherches ultérieures sont essentielles pour répondre à l'interrogation suivante : se pourrait-il que les approches pédagogiques utilisées par les concepteurs de formations à distance évoluent avec les avancées technologiques? Le cas échéant, il serait difficile de départager l'effet des avancées technologiques des effets pédagogiques, comme l'ont souligné à plusieurs reprises Russell (2001) et, plus récemment, Fendler et al. (2018). Par ailleurs, d'autres investigations apparaissent nécessaires afin de vérifier si l'efficacité de la FAD diffère avec les avancées technologiques, selon que les indicateurs concernent l'efficacité individuelle ou institutionnelle. Dans la première situation, rappelons-le, l'usager se responsabilise quant à l'atteinte ou non de ses buts, et dans la seconde, le concepteur respecte les objectifs désignés par l'institution (Pourcelot et Abid-Zarrouk, 2015). De même, des recherches additionnelles visant à examiner si l'adoption des technologies a suivi une approche ascendante ou descendante devraient être entreprises. D'après Abid-Zarrouk, la première « suppose que l'intégration vient d'en bas, en l'occurrence des enseignants [et] la seconde repose sur le fait que l'intégration est décidée par les pouvoirs centraux et est ainsi imposée à l'ensemble du système éducatif et par conséquent aux enseignants à travers de grands plans nationaux » (2012b, p. 3). La question de recherche se formulerait comme suit : dans les cas où la FAD semble différer selon les avancées technologiques, l'adoption des nouvelles technologies a-t-elle été dictée par l'établissement ou choisie par les professeurs et les concepteurs de cours? 


\section{Conclusion}

L'efficacité de la FAD diffère selon les avancées technologiques. Ce constat est appuyé par 16 méta-analyses, comprenant 862 études primaires menées chacune à l'aide d'un schéma de recherche rigoureux et comptant plus de 200000 participants en provenance des continents américain (incluant le Canada), asiatique, européen et océanien. De fait, avec un indicateur d'une valeur $\geq 0,20$, le pourcentage d'effets d'ampleur est plus grand avec les ODE (44\%) qu'avec les PDE/ODE (29\%) et les PDE (o \%). De même, avec un indicateur d'une valeur $\geq 0,40$, le pourcentage d'effets d'ampleur est également plus grand avec les ODE (31\%) qu'avec les PDE/ODE (o \%) et les PDE (o \%). Ces résultats permettent une meilleure compréhension de l'efficacité de la FAD selon les avancées technologiques, mais ils ne permettent en rien d'affirmer qu'elles sont une panacée, que leur utilisation correspond nécessairement à une réussite de l'apprentissage, ou encore, que le rôle des acteurs dans la FAD n'est pas central. Notre contribution consiste à mettre en lumière une réalité basée sur un vaste ensemble d'études validées, que le monde de l'éducation doit désormais prendre en considération. Il est ainsi à prévoir que la FAD sera un élément clé de plus en plus crucial dans l'avenir de la formation postsecondaire canadienne et internationale (Lee, 2017; Simard, 2018).

Selon les résultats de notre méga-analyse, une attention particulière devrait être accordée à l'intégration des nouvelles technologies dans l'enseignement supérieur. D'ailleurs, des études subséquentes devraient davantage viser la détermination des variables qui interviennent pour améliorer l'efficacité de la FAD postsecondaire en lien avec l'utilisation de ces nouvelles technologies. Plus les recherches permettront de préciser ces variables, plus l'internationalisation des institutions canadiennes postsecondaires deviendra une réalité notable et, surtout, plus l'accessibilité de l'enseignement supérieur pour les citoyens du pays s'avérera une valeur ajoutée essentielle.

\section{Notes}

1. L'abréviation « PDE » et l'abréviation « ODE » (plus loin), qui réfèrent à des expressions de langue anglaise, ont été retenues étant donné leur utilisation très répandue dans les écrits nationaux et internationaux.

\section{References}

Abid-Zarrouk, S. B. (2015). Les efficacités technologiques éducatives. Dans S. B. AbidZarrouk (dir.), Estimer l'efficacité en éducation (p. 215-228). Paris : L'Harmattan.

Abid-Zarrouk, S. B. (2013). Efficacité de l'enseignement à distance : mythe ou réalité? Paris : L'Harmattan.

Abid-Zarrouk, S. B. (2012a). La question de l'efficacité des forums virtuels sur la réussite. Questions Vives, 6(16), 1-13. doi:10.4000/questionsvives.941

Abid-Zarrouk, S. B. (2012b). Innovations pédagogiques, incertitude et biais cognitifs. Recherches \& Éducations, 6, 55-70.

Abid-Zarrouk, S. B. (2010a). L'abandon : facteur d'inefficacité de l'enseignement en ligne. Sciences et Technologies de l'e'Éducation et la Formation, 17, 103-124.

Adnan, M., Kalilioglu, F., Gulbahar, A. M. K. et Brunner, B. R. (2017). Engaged learning 
through online collaborative public relations projects across universities. Journalism \& Mass Communication Educator, 72(4), 442-460. doi:10.1177/1077695816686440

Allen, M., Mabry, E., Mattrey, M., Bourhis, J., Titsworth, S. et Burrell, N. (2004). Evaluating the effectiveness of distance learning: A comparison using meta-analysis. Journal of Communication, 54(3), 402-420.

Anderson, W. (2013). Independent learning. Dans M. G. Moore (dir.), Handbook of distance education (p. 86-103). New York, NY : Routledge.

Banerjee, S. et Firtella, J. (2017). Pedagogical models for enhancing the cross-cultural online public health learning environment. Health Education Journal, 76(5), 622-631. doi:10.1177/0017896917710970

Bates, T. (2018). The 2017 national survey of online learning in Canadian postsecondary education: Methodology and results. International Journal of Educational Technology in Higher Education, 15(29), 1-17.

Bernard, R. M., Abrami, P. C., Lou, Y., Borokhovski, E., Wade, A., Wozney, L., Wallet, P. A., Fiset, M. et Huang, B. (2004). How does distance education compare to classroom instruction? A meta-analysis of the empirical literature. Review of Educational Research, 74(3), 379-439.

Bissonnette, S., Richard, M., Gauthier, C. et Bouchard, C. (2010). Quelles sont les stratégies d'enseignement efficaces favorisant les apprentissages fondamentaux auprès des élèves en difficulté de niveau élémentaire? Résultats d'une méga-analyse. Revue de recherche appliquée sur l'apprentissage, 3(1), 1-35.

Borenstein, M. et Tanner-Smith, E. E. (2016). Introduction to systematic review and meta-analysis methods. Repéré à http://campbellcollaboration.org

Bozkurt, A., Yazıcı, M. et Aydın, I. E. (2018). Cultural diversity and its implications in online networked learning spaces. Dans E. G. Kumtepe et N. S. Ersoy (dir.), Interaction in distance education: A cultural critique of instructional dialogue (p. 56-82). Hershey, PA: IGI Global. doi:10.4018/978-1-5225-3076-3

Bozkurt, A., Akgun-Ozbek, E., Yilmazel, S., Erdogdu, E., Ucar, H., Guler, E., Sezgin, S., Karadeniz, A., Sen-Ersoy, N., Goksel-Canbek, N., Dincer, G. D., Ari, S. et Aydin, C. H. (2015). Trends in distance education research: A content analysis of journals 2009-2013. International Review of Research in Open and Distributed Learning, 16(1), 330-363.

Carter, C. S., Solberg, L. B. et Solberg, L. M. (2017). Applying theories of adult learning in developing online programs in gerontology. Journal of Adult and Continuing Education, 23(2), 197-205. doi:10.1177/1477971417721718

Chang, M. M. et Lin, M. C. (2013). Strategy-oriented web-based English instruction: A meta-analysis. Australasian Journal of Educational Technology, 29(2), 203-211.

Clark, R. E. (2008). Évaluer l'enseignement à distance : stratégies et avertissements. Distances et Savoirs, 1(7), 93-112.

Collin, S., Guichon, N. et Ntébutsé, J.-G. (2015). Une approche critique des usages numériques en éducation. STICEF, 22, 89-117. 
Cook, D. A., Levinson, A. J., Gardside, S., Dupras, D. M., Erwin, P. J. et Monton, V. M. (2010a). Instructional design variations in internet-based learning for health professions education: A systematic review and meta-analysis. Academic Medicine, 85(5), 909-922.

Cook, D. A., Levinson, A. J. et Garside, S. (2010b). Time and learning efficiency in Internet-based learning: A systematic review and meta-analysis. Advances in Health Sciences Education, 15(5), 755-770.

Cook, D. A., Levinson, A. J., Garside, S., Dupras, D. M., Erwin, P. J. et Montori, V. M. (2008). Internet-based learning in the health professions: A meta-analysis. Journal of the American Medical Association, 300(10), 1181-1196.

Cui, D., Arthur, N. et Domene, J. F. (2017). Accompanying partners of international students: Reflections on three issues. Canadian Journal of Higher Education, 47(1), 171190.

Darabi, A., Liang, X., Suryavanshi, R. et Yurekli, H. (2013). Effectiveness of online discussion strategies: A meta-analysis. The American Journal of Distance Education, 27, 228-241.

Ellis, A. K. (2005). Research on educational innovations. Larchmont, NY : Eye on education.

Fendler, R. J., Ruff, C. et Shrikhande, M. M. (2018). No significant difference. Online Learning, 22(1), 39-60.

Fortin, M. F. et Gagnon, J. (2015). Fondements et étapes du processus de recherche : méthodes quantitatives et qualitatives. Montréal, QC : Chenelière Éducation.

Garrison, D. R. et Akyol, Z. (2013). The community of inquiry theoretical framework. Dans M. G. Moore (dir.), Handbook of Distance Education (p. 104-119). New York, NY : Routledge.

Günes, A. et Bozna, H. (2018). A futurist approach for open and distance 21st century learners. Dans E. G. Kumtepe et N. S. Ersoy (dir.), Interaction in distance education: A cultural critique of instructional dialogue (p. 281-291). Hershey, PA: IGI Global. doi:10.4018/978-1-5225-3076-3

Gurevitch, J., Koricheva, J., Nakagawa, S. et Stewart, G. (2018). Meta-analysis and the science of research. Nature, 555, 175-182. doi:10.1038/nature25753

Hattie, J. (2015). Teacher-ready research review: The applicability of visible learning to higher education. Scholarship of Teaching and Learning in Psychology, 1(1), 79-91.

Hsu, Y. C., Chibng, Y. H. et Grabowski, B. L. (2014). Web 2.0 applications and practices for learning through collaboration. Dans J. M. Spector et al. (dir.), Handbook of research on educational communications and technology (p. 747-758). New York, NY : Springer Science+Business. doi:10.1007/978-1-4614-3185-5_30

Jahng, N., Krug, D. et Zhang, Z. (2007). Student achievement in online education compared to face-to-face education: A meta-analysis. European Journal of Open, Distance and E-learning, 1, 1-20. http://www.eurodl.org/?p=archives\&year=2007\&hal fyear $=1 \&$ article $=253$ 
Johnson, S. (2014). Applying the seven principles of good practice: Technology as a lever in an online research course. Journal of Interactive Online Learning, 13(2), 41-50.

Kesim, M. (2018). Culture, technology and education in the digital age. Dans E. G. Kumtepe et N. S. Ersoy (dir.), Interaction in distance education: A cultural critique of instructional dialogue (p. 83-105). Hershey, PA: IGI Global. doi:10.4018/978-1-52253076-3

Khadimally, S. (2018). Evaluating trends and issues in distance learning. Dans E. G. Kumtepe et N. S. Ersoy (dir.), Interaction in distance education: A cultural critique of instructional dialogue (p. 130-145). Hershey, PA: IGI Global. doi:10.4018/978-1-52253076-3

Kumtepe, E. G. et Ersoy, N. S. (2018). Interaction in distance education: A cultural critique of instructional dialogue. Dans E. G. Kumtepe et N. S. Ersoy (dir.), Interaction in distance education: A cultural critique of instructional dialogue (p. 222-248). Hershey, PA: IGI Global. doi:10.4018/978-1-5225-3076-3

Landers, R. N. et Reddock, C. M. (2016). A meta-analytic investigation of objective learner control in web-based instruction. Journal of Business and Psychology. 32(4), 455-478. doi:10.1007/s10869-016-9452-y

Lee, K. (2017). Rethinking the accessibility of online higher education: A historical review. Internet and Higher Education, 33, 15-23.

Lou, Y., Bernard, R. M. et Abrami, P. C. (2006). Media and pedagogy in undergraduate distance education: A theory-based meta-analysis of empirical literature. Educational Technology Research and Development, 54, 141-176. doi:10.1007/s11423-006-852-x

Machtmes, K. et Asher, J. W. (2000). A meta-analysis of the effectiveness of telecourses in distance education. American Journal of Distance Education, 14(1), 2746. doi:10.1080/08923640009527043

Martin, F., Ahlgrim-Delzell, L. et Budhrani, K. (2017). Systematic review of two decades (1995 to 2014) of research on synchronous online learning. The American Journal of Distance Education, 31(1), 3-19. http://dx.doi.org/10.1080/08923647.2017.1264807

Means, B., Toyama, Y., Murphy, R., Bakia, M. et Jones, K. (2010). Evaluation of evidence-based practices in online learning: A meta-analysis and review of online learning studies. Washington, DC : U.S. Department of Education, Office of Planning, Evaluation, and Policy Development, Policy and Program Studies Service Center for Technology in Learning.

Merrill, M. D. et Elen, J. (2014). A look forward. Dans J. M. Spector et al.. (dir.), Handbook of research on educational communications and technology (p. 873-874). New York, NY : Springer Science+Business. doi:10.1007/978-1-4614-3185-5_30

Moore, M. G. (2013). The theory of transactional distance. Dans M. G. Moore (dir.), Handbook of distance education (p. 66-85). New York, NY : Routledge.

Pourcelot, C. et Abid-Zarrouk, S. B. (2015). Construire des outils d'évaluation de l'efficacité d'un dispositif pédagogique à distance : le cas de l'efficacité interne d'IFADEM RDC/Katanga. Distances et médiations des savoirs, 3(11), 1-23. doi:10.4000/dms.1099 
Richardson, J. C., Maeda, Y., Lv, J. et Caskurlu, S. (2017). Social presence in relation to students' satisfaction and learning in the online environment: A meta-analysis. Computer in Human Behavior, 71, 402-417.

Roberts, R. M. (2011). Best instructional practices for distance education: A metaanalysis (Thèse de doctorat). Accessible par ProQuest Dissertations \& Theses. (3475479). Repéré à https://digitalscholarship.unlv.edu/thesesdissertations/1240/

Russell, T. L. (2001). The no significant difference phenomenon: A comparative research annotated bibliography on technology for distance education. Repéré à http:// www.nosignificantdifference.org

Saba, F. (2013). Building the future: A theoretical perspective. Dans M. G. Moore (dir.), Handbook of distance education (p. 49-65). New York, NY : Routledge.

Schmidt, F. L. et Hunter, J. E. (2015). Methods of meta-analysis. Thousand Oaks, CA : Sage.

Shachar, M. et Neumann, Y. (2003). Differences between traditional and distance education academic performances: A meta-analytical approach. International Review of Research in Open and Distance Education, 4(2), 1-20.

Simard, Y. (2018). L'efficacité de la formation à distance au niveau postsecondaire : une méga-analyse (Thèse de doctorat non publiée). Université Laval, Québec.

Sitzmann, T., Kraiger, K., Stewart, D. et Wisher, R. (2006). The comparative effectiveness of web-based and classroom instruction: A meta-analysis. Personnel Psychology, 59(3), 623-664. doi:10.1111/j.1744-6570.2006.00049.x

Sung, Y. T., Chang, K. E. et Liu, T. C. (2016). The effects of integrating mobile devices with teaching and learning on students' learning performance: A meta-analysis and research synthesis. Computers \& Education, 94, 252-275.

Sung, Y. J., Schwander, K., Arnett, D. K., Kardia, S. L. R., Rankinen, T., Bouchard, C., Boerwinkle, E., Hunt, S. C. et Rao, D. C. (2014). An empirical comparison of meta-analysis and mega-analysis of individual participant data for identifying gene-environment interactions. Genetic Epidemiology, 38(4), 369-378.

Van der Kleij, F. M., Feskens, R. C. W. et Eggen, T. J. H. (2015). Effects of feedback in a computer-based learning environment on students' learning outcomes: A meta-analysis. Review of Educational Research, 85(4), 475-511. doi:10.3102/0034654314564881

Williams, S. L. (2006). The effectiveness of distance education in allied health science programs: A meta-analysis of outcomes. American Journal of Distance Education, 2O(3), 127-141.

Zancaro, A., Nunes, C. S. et Domingues, M. J. C. (2017). Evaluation of free platforms for delivery of massive open online courses (MOOCs). Turkish Online Journal of Distance Education, 18(1), 166-181.

Zhao, Y., Lei, J., Yan, B., Lai, C. et Tan, H. S. (2005). What makes the difference? A practical analysis of research on the effectiveness of distance education. Teachers College Record, 107(8), 1836-1884. 
Zheng, L. (2016). The effectiveness of self-regulated learning scaffolds on academic performance in computer-based learning environments: A meta-analysis. Asia Pacific Education Review, 117(2), 187-202. doi:10.1007/s12564-016-9426-9

Ziegler, N. (2016). Synchronous computer-mediated communication and interaction a meta-analysis. Studies in Second Language Acquisition, 38, 553-586.

\section{Notices biographiques}

Yanik Simard

Université TÉLUQ

simard.yanik@univ.teluq.ca

Yanik Simard est détenteur d'un doctorat en philosophie (Ph. D.), d'un doctorat en psychopédagogie (Ph. D.) et d'une maîtrise en administration publique. Il a été professionnel de recherche à l'Université Laval, à l'Université TÉLUQ, à l'École d'administration publique (ENAP), au ministère de l'Éducation et de l'Enseignement supérieur (MEES) et au Cégep de Sainte-Foy. Il est actuellement postdoctorant à l'Université TÉLUQ, où il effectue des méga-analyses sur l'efficacité des jeux électroniques éducatifs concernant diverses populations. Il est le premier auteur ou le coauteur d'une vingtaine de publications liées notamment à l'éducation des adultes, à la formation à distance et à l'éducation en santé.

Clermont Gauthier, Ph. D., est professeur titulaire à l'Université Laval. Il est cofondateur et chercheur régulier du Centre de recherche interuniversitaire sur la formation et la profession enseignante (CRIFPE). Il a publié, seul ou en collaboration, plus de quarante ouvrages et une centaine d'articles, et il a prononcé plus de 300 communications nationales et internationales. Il a été titulaire d'une chaire de recherche du Canada sur l'étude de la formation à l'enseignement et élu membre de la Société royale du Canada.

Mario Richard est professeur titulaire au Département d'éducation de l'Université TÉLUQ et chercheur associé au Centre de recherche et d'intervention sur la formation et la profession enseignante (CRIFPE). Il a offert plus de 125 communications portant sur le développement professionnel des intervenants scolaires dans divers congrès et colloques en éducation et a participé à la rédaction de plus de 50 publications sur le thème de l'efficacité de l'enseignement et des écoles. Ses travaux actuels portent sur le téléapprentissage ainsi que sur le développement professionnel et l'accompagnement des intervenants en éducation à partir des recherches en efficacité de l'enseignement. 